\title{
Word concreteness and encoding effects on context-dependent discrimination
}

\author{
ANA M. FRANCO-WATKINS and MICHAEL R. DOUGHERTY \\ University of Maryland, College Park, Maryland
}

\begin{abstract}
In three experiments, we investigated the effects of word concreteness and encoding instructions on context-dependent discrimination in verbal contexts, using Murnane, Phelps, and Malmberg's (1999) ICE (item, context, ensemble) theory as the framework. Word concreteness was manipulated within participants, and encoding was manipulated between participants. It was hypothesized that the magnitude of context-dependent discrimination would be affected by both concreteness and encoding instructions. Imagery instructions resulted in context-dependent discrimination for both concrete and abstract word pairs across all the experiments. Context-dependent discrimination was observed under rote instructions for concrete and abstract words, particularly when the same context word was paired multiple times with the targets. The results indicated that context-dependent discrimination is not dependent solely on the use of interactive imagery instructions or on word concreteness.
\end{abstract}

A fundamental topic in memory research concerns the role of context on memory performance. Of particular interest is the degree to which the similarity between the contexts in which the information was first encoded and later retrieved affects retrieval performance. In the typical paradigm, participants study a list of words within a particular context and are tested in either the same context or a different context. The typical finding, at least for recall, is that memory performance is better when study and test take place in the same context, relative to a different context (Smith, 1988; Tulving \& Thomson, 1973). However, inconsistent context effects (CEs) occur for recognition (Murnane, Phelps, \& Malmberg, 1999; Smith, 1988).

The inconsistencies observed for recognition can be further distinguished on the basis of how the term context is defined. One can distinguish between two general classifications of the context: environmental and verbal contexts (i.e., global and local contexts, Dalton, 1993; context alpha and context beta, Wickens, 1987). Environmental context can refer to moods, backgrounds, ${ }^{1}$ test locations, and so forth, whereas verbal context refers to using another word as the context, as in a word pair paradigm

This research was supported by funds provided by the Department of Psychology, University of Maryland. The authors are grateful to Kevin Murnane for his suggestions for conducting Experiment 3 and to William Hockley, Kevin Murnane, John Wixted, and an anonymous reviewer for comments on an earlier version of the manuscript. We also thank Fran Drummond for her assistance with data collection. Correspondence concerning this article should be addressed to A. M. FrancoWatkins, Department of Psychology, Auburn University, Auburn, AL 36849, or M. R. Dougherty, Department of Psychology, University of Maryland, College Park, MD 20742 (e-mail: afrancowatkins@auburn .edu or mdougherty@psyc.umd.edu).

Note-This article was accepted by the previous editorial team, when Colin M. MacLeod was Editor.
(Dalton, 1993; Smith, 1988; Wickens, 1987). Both types of CEs have been investigated in the literature. In a metaanalysis of incidental environmental CEs, Smith and Vela (2001) revealed that environmental context had a reliable effect on context-dependent recognition (weighted $d=$ .27). However, although Smith and Vela's meta-analysis revealed an overall effect of context on recognition, several studies have failed to show reliable CEs (e.g., Godden \& Baddeley, 1975; Smith, Glenberg, \& Bjork, 1978). In contrast to the inconsistent findings regarding environmental CEs, verbal CEs have been reliably observed (Dalton, 1993).

Using a verbal context paradigm, Light and CarterSobell (1970) demonstrated that when homographic nouns differed in semantic interpretation from study to test, recognition performance declined substantially. They assumed that the change in context interfered with the retrieval of the semantic properties of the word. Similar findings have been reported by a number of researchers, who have shown that changes in verbal context reduce recognition in word pairs (Bradley \& Glenberg, 1983; Humphreys, 1976; Tulving \& Thomson, 1973).

The findings above on CEs in recognition have prompted several researchers to explore the reasons underlying the inconsistencies in context effects. One factor that seems to be crucial is the encoding conditions. For example, dividing attention at encoding has a greater impact on eventual retrieval (for both recognition and recall) than does dividing attention during retrieval (Craik, Govoni, NavehBenjamin, \& Anderson, 1996; Craik, Naveh-Benjamin, Ishaik, \& Anderson, 2000). In one recent analysis, Murnane et al. (1999) argued that the nature of the CE depends on how participants use context information during encoding. In several experiments reported by Murnane and Phelps (1993, 1994, 1995), in which the location of the item on the screen and the color were used as the con- 
text, only same-direction effects on both the hit rate (HR) and the false alarm rate (FAR) were observed, with no net effect on discrimination (operationalized in terms of $d^{\prime}$ ) between same and different contexts. However, when the context contained rich visual stimuli, there was a reliable effect on $d^{\prime}$ (Murnane et al., 1999). Thus, it is possible to detect a change in recognition (HR and FAR) based on the context between learning and test, without necessarily revealing changes in discrimination (as measured by HR minus FAR or $d^{\prime}$ ).

The two CEs alluded to above (i.e., same-direction effects on HR and FAR vs. an effect on $d^{\prime}$ ) can be classified as two different types of CEs (Murnane et al., 1999). Context-dependent recognition refers to any change in recognition that depends on whether the context at test is the same as or different from the context at learning. Typically, context-dependent recognition is characterized by higher HRs and FARs in the same-context condition than in the different-context condition. However, discrimination, as indexed by $d^{\prime}$, might not be affected if the changes in both the HR and the FAR for same and different conditions offset one another. For example, if the changes in the HR and the FAR are roughly parallel, $d^{\prime}$ will be unchanged. Context-dependent discrimination is a special case of context-dependent recognition, in which the ability to discriminate between targets and distractors is affected by the context used during test. Typically, context-dependent discrimination is characterized by a greater increase in the HR, relative to the increase in the FAR, which in turn results in a higher $d^{\prime}$ for the samecontext than for the different-context condition.

To account for different types of environmental CEs in recognition, Murnane et al. (1999) proposed a generalized global-matching model called ICE. According to ICE, traces in memory can consist of three types of information: item (I) information, which corresponds to the to-be-remembered item; associated context (C) information, which corresponds to the context present at the time of encoding; and an ensemble (E), which corresponds to the integration of the item and the context information. The ensemble is a theoretical entity that may or may not be stored alongside the item and context information, depending on how the context is treated at encoding. For example, suppose that a participant studies a list of words and each word (e.g., apple) is presented on one of several backgrounds (e.g., a school bus). In this case, the item would consist of the word apple, and the associated context would be the school bus background. An ensemble could be created if the participant integrated the word apple with the image of the bus. For instance, the participant might form a mental image of large red apples seated on the bus. ICE does not specify the nature of how integration might be achieved (e.g., through imagery or semantic relatedness or phonologically). However, Murnane et al. assumed that ensembles are formed when participants are instructed to form a unique integration of item and context information. The ensemble does not necessitate that the integration of the item and the context be achieved via visual imagery. Nonetheless, most research that we are aware of that has tested ICE theory has manipulated ensemble formation by instructing participants to form an interactive image between the item and the context (e.g., a picture or background; Murnane et al., 1999).

ICE assumes a global memory matching process, in which the retrieval cue or the probe is matched against all the items stored in memory. ${ }^{2}$ As a result, several predictions fall out of the ICE framework, depending on how the items are encoded and on the contexts available at encoding and retrieval (see Murnane et al., 1999, for equations used to derive predictions). Table 1 , which is based on Murnane et al.'s Table 3 (1999, p. 408), presents ICE's predictions regarding CEs, depending on whether item, associated context, and ensemble information are stored in memory. In the description of ICE predictions, the first column will be ignored; we shall return to this column when deriving the hypotheses for the experiments presented in this article.

The first row of Table 1 represents the case in which context information is not stored with the item information in memory. Because context information is absent from memory, there should be no effect of context present at retrieval on the HR, FAR, and $d^{\prime}$. The next row represents the case in which an item and an associated context are stored in memory without the formation of an ensemble. ICE predicts a greater HR and FAR in same-context tests than in different-context tests, with no net effect of context on $d^{\prime}$. For the HR, match strength will be greater when an item and an associated context (e.g., a target item in same context at test) are used to match memory than when an item and a new context (e.g., a target item with a

Table 1

ICE Theory Predictions for Context Effects (CEs) Based on Item, Associated Context, and Ensemble Information

\begin{tabular}{|c|c|c|c|c|c|}
\hline \multirow[b]{2}{*}{ Encoding and Context Manipulation } & \multicolumn{2}{|c|}{ Information } & \multicolumn{3}{|c|}{ CE (Same-Different) } \\
\hline & $\begin{array}{c}\text { Associated } \\
\text { Context }\end{array}$ & Ensemble & HR & FAR & $d^{\prime}$ \\
\hline Item only & - & - & $\times$ & $\times$ & $\times$ \\
\hline Unique or multiple contexts: Rote conditions & + & - & $\checkmark$ & $\checkmark$ & $\times ?$ \\
\hline Unique contexts: Imagery (rote?) conditions & - & + & $\checkmark$ & $\times$ & $\checkmark$ \\
\hline Multiple contexts: Imagery conditions & + & + & $\boldsymbol{V}$ & $\checkmark$ & $\checkmark ?$ \\
\hline
\end{tabular}


different context at test) are used. Similarly, for the FAR, a new item presented with an old associated context (e.g., a distractor with an old associated context at test) will elicit greater match strength than when both the item and the context are new (e.g., a distractor with a new context at test). Thus, if only an item and an associative context are stored at study, context-dependent discrimination is unlikely to occur, because match strength increases for both the HR and the FAR in the same-context condition. ${ }^{3}$

The third row represents the case in which only ensemble information is stored in memory. In this case, ICE predicts greater increases in the HR than in the FAR in same-context conditions than in different-context conditions. The ensemble information is crucial to the added advantage of the increase in HR for same-context conditions because the ensemble information can contribute to match strength only when it is present both in memory and in the test probe, and this is possible only for targets tested in the same-context condition. Thus, context-dependent discrimination is predicted to occur only when item and context information are integrated into an ensemble at encoding and subsequently used at retrieval (Bayen, Phelps, \& Spaniol, 2000; Murnane et al., 1999). However, when an ensemble is stored alongside the item and associated context, as indicated in the last row of Table 1, there is an increase in both the HR and the FAR. Nonetheless, it is possible to have context-dependent discrimination if the increases in the HR are greater than those in the FAR for the same-context condition than for the different-context condition.

The formation of different types of ensembles may be produced by different types of encoding instructions. For example, imagery encoding may prompt the formation of image-based ensembles (i.e., ensembles rich in visual details). Because image-based ensembles depend, to some extent, on the richness of the visual features, one might expect image-based ensembles to be dependent on the concreteness or imaginability of the to-be-remembered items. Thus, for imagery-based encoding, context-dependent discrimination should be larger when the to-be-remembered items are concrete or highly imaginable than when the items are abstract or less imaginable.

In contrast, one might expect semantic ensembles to be formed if the encoding instructions encourage semantic processing or phonologically based ensembles when phonology is emphasized at encoding. In these cases, ensembles are assumed to be based on nonvisual features, and hence, their formation should be unrelated to the concreteness or imaginability of the to-be-remembered items. Thus, for rote rehearsal encoding (i.e., repeating a word pair), context-dependent discrimination should be unaffected by word concreteness. ${ }^{4}$

Crucial for a comprehensive theory of CEs is the ability both to include general definitions of context and to handle encoding and retrieval conditions. Although the ICE theory was formulated to explain environmental CEs, one could presumably use its predictions to examine verbal CEs. In this way, the ICE model might provide a framework for understanding both encoding and retrieval components involved in recognition memory. A word pair paradigm would be most appropriate to examine ICE predictions for verbal context, because one word can be designated as the item information and the other word as the context information.

The purpose of the present experiments was to apply ICE to verbal context conditions, as well as to examine the robustness of ICE's predictions in regard to word concreteness and encoding manipulations. First, we were interested in whether the magnitude of the contextdependent effects would be dependent on the concreteness of the to-be-remembered information. Thus, our research examined whether concreteness affects context-dependent discrimination under encoding instructions that either facilitate or do not facilitate the formation of ensembles. Previous research on word concreteness has shown better recognition performance for concrete words than for abstract words (Glanzer \& Adams, 1990; Glanzer, Adams, Iverson, \& Kim, 1993), and this finding was extended to discrimination, where discrimination was greater for concrete words than for abstract words (Glanzer \& Adams, 1990). However, the relative effectiveness of contextdependent discrimination for concrete and abstract words has not been investigated within the framework of ICE. Is the presence of concrete word pairs sufficient to produce context-dependent discrimination, or is integration of item and context information necessary for context-dependent discrimination to occur?

Second, we were interested in whether the method of encoding would influence the occurrence of contextdependent discrimination. Murnane et al. (1999) argued that integration of an item and associated context into an ensemble plays an important role in promoting contextdependent discrimination effects. As a result, contextdependent discrimination should be more pronounced when participants are given instructions that facilitate item-context integration, such as telling participants to form an interactive imagery that combines the item and the context, relative to instructions that promote rote rehearsal, such as instructing participants to repeat a word pair over and over. Moreover, previous research has suggested that the success of rehearsal strategies in improving memory performance is sensitive to word concreteness. For example, Richardson (1998) described how imaginal mediators (e.g., a mental image combining two words in a word pair) were more effective for learning concrete nouns and verbal mediators (e.g., phonological similarity) for learning abstract materials. Although imagery instructions are effective for both concrete and abstract pairs, there are stronger effects for concrete pairs than for $a b-$ stract pairs (Groninger \& Groninger, 1982; Richardson, 1998). Consequently, we predicted that imagery would be more efficacious for concrete words than for abstract words. Thus, we predicted that context would interact with encoding strategy and word type.

The experiments reported by Murnane et al. (1999) used words embedded in a rich visual context (e.g., a picture of a classroom with a blackboard). Because of this, we wanted to examine whether the use of a concrete vi- 
sual environmental context was a critical feature of their experimental design. Does the magnitude of the CE depend on how well one can visualize the integration of item and context information? Given that concrete words are easier to integrate into an ensemble than abstract words, particularly under imagery instructions, we predicted that context-dependent discrimination, as reflected in $d^{\prime}$ for same and different contexts, would be greater for concrete words than for abstract words.

Our primary hypotheses concerned whether contextdependent discrimination would be differentially affected by word concreteness and encoding instructions. In the experiments that follow, we used word pairs as stimuli, with one word specifically indicated to the participants as the to-be-remembered item and the other as context information. In Experiments 1 and 2, a unique context word was associated with each item, whereas the same context word was associated with multiple to-be-remembered items in Experiment 3.

For all the experiments, we manipulated encoding by varying instructions that emphasized the use of context information. In the interactive imagery condition, the participants were instructed to form an interactive image that contained both the item and the context. For Experiments 1 and 2, this condition is represented by the third row of Table 1, where only ensemble information is used. We assumed that when a unique context word is used with each item presentation, imagery instructions should promote the formation of ensemble information. We expected this condition to show the highest amount of contextdependent discrimination. Interactive imagery instructions should increase the HR to a larger extent than the FAR for same-context conditions, which, in turn, should result in greater discriminability for the same-context conditions than for the different-context conditions.

All three experiments included a rote rehearsal condition as a comparison condition, in which the participants were to continually repeat the word pair while it remained on the computer screen. Our assumption was that the rote condition would not induce the formation of ensembles; therefore, it would be represented by the second row of Table 1. In this condition, context-dependent discrimination is unlikely to occur. We expected to observe contextdependent recognition in the rote condition for both the HR and the FAR, since in the rote condition participants presumably use the item and the associated context at encoding, as per instructions. However, it is conceivable that rote rehearsal instructions might promote some sort of non-imagery-based integration (e.g., phonological, syntactic, or semantic integration), which might result in context-dependent discrimination. There is some evidence that words can be based on relational (Humphreys, 1976), semantic (Light \& Carter-Sobell, 1970), or maintenance (Bradley \& Glenberg, 1983) properties. If this is the case, the rote condition would correspond to the third row of Table 1, resulting in effects similar to those in the imagery condition. However, we expected the magnitude of the CEs to be greater in the imagery than in the rote condition, particularly for concrete words. Moreover, if the rote re- hearsal condition resulted in context-dependent discrimination, there should be no effect of word concreteness on the magnitude of the $\mathrm{CE}$, because one would presume that it would be based on a phonological or semantic integration, rather than on an image-based integration.

In addition, Experiments 1 and 2 included a condition in which the participants were told to focus on the item only (item-only instructions). This condition, which serves as the control condition, is represented by the first row of Table 1, where no CEs should be observed. Thus, we predicted the following pattern for context-dependent discrimination: imagery $>$ rote $\geq$ item only, which constitutes a two-way interaction between context and encoding.

As was mentioned above, we also manipulated word concreteness. We hypothesized that word concreteness would affect the magnitude of context-dependent discrimination, since concrete words should be more easily integrated into an ensemble than are abstract words. Thus, we predicted that concreteness would interact with encoding instructions. Specifically, isolation of the imagery condition should reveal greater context-dependent discrimination effects for concrete words than for abstract words. The effectiveness of the imagery instructions should depend, to some extent, on the concreteness of the to-be-remembered items, since it is easier to conjure up images for concrete words. In the rote rehearsal condition, the participants were told to repeat both words subvocally for the duration they were presented on the screen, and in the item-only condition, the participants were told to repeat (to themselves) the item information once. On the basis of ICE theory, contextdependent discrimination should be unlikely in the latter two conditions because neither promotes the formation of ensembles based on encoding instructions. However, if nonvisual integration is feasible in the rote condition, we would expect that word concreteness should not affect the magnitude of context-dependent discrimination.

\section{EXPERIMENT 1}

\section{Method}

\section{Participants}

Ninety-four undergraduates at the University of Maryland participated in the experiment in order to fulfill partial course requirements.

\section{Materials}

All the materials were presented on a computer screen. Both study and test lists consisted of abstract and concrete word pairs. Abstract and concrete words were selected from the Toronto Word Pool (Friendly, Franklin, Hoffman, \& Rubin, 1982) on the basis of concreteness ratings ranging from 1 (abstract) to 7 (concrete). Words rated from 1 to 3 were selected as abstract words, and words rated from 5 to 7 were considered concrete words. Two words appeared in the center of the screen, with the left word in green text (item word) and the right word in black text (context word). The words were displayed on the same row within 3 in. of one another. All the words were presented in 28-point font. The pairs were always presented as an intact pair in terms of concreteness. Specifically, an abstract item was always paired with an abstract context word (e.g., mental virtue), and a concrete item was paired with a concrete context word (e.g., tunnel kitten). Each word pair was randomly generated from a list of 265 abstract words and 350 concrete words so that there was a unique pairing of words for each participant. 


\section{Design and Procedure}

The experiment consisted of a 2 (concreteness: abstract or concrete) $\times 3$ (encoding: rote, imagery, or item only) $\times 2$ (test context: same or different) design. Both concreteness and test context were manipulated within participants, and encoding was manipulated between participants. The experiment involved a study and a test phase, with a brief intervening reasoning task between phases that lasted approximately $5 \mathrm{~min}$.

Study phase. The participants viewed a study list containing 80 word pairs ( 40 concrete and 40 abstract pairs), which were randomly presented for $3 \mathrm{sec}$ on the computer screen, with a 0.5 -sec delay between word pairs. Each participant received a random presentation of all the word pairs.

In addition, the participants were randomly assigned to one of three encoding instruction conditions (imagery, rote, or item only). In the imagery condition, the participants were instructed that they should integrate both words into a vivid image. For instance, if the word pair "lion-paper" occurred, one could think of a string of paper lions blowing on a clothesline. In the rote condition, the participants were instructed to repeat each word in the pair over and over (subvocally) until the words disappeared from the screen. In the item condition, the participants were instructed to repeat once only the word in green. ${ }^{5}$ Of particular note is that all the participants were instructed that their memory for the words that appeared in green would be tested at a later time during the experiment. However, the participants in the imagery and rote conditions were instructed that forming an interactive image and repeating the word pair, respectively, would enhance their ability to remember the word that appeared in green. Thus, if the participants ignored the interactive imagery or rote rehearsal encoding instructions, context effects should not occur.

Test phase. The participants were randomly presented with 160 word pairs. As with the study list, the left word of the pair was presented in green, and the right word of the pair was presented in black text. The participants were asked to determine whether the word appearing in green had been on the study list. If the word had been on the list, they were instructed to press the "T" key on the keyboard, and if the word had not been on the study list, they were instructed to press the "F" key. In order to create the test list that contained both targets and distractors in same and different contexts, test word pairs were distributed into the following categories: 40 target same, 40 target different, 40 distractor same, and 40 distractor different. Target-same word pairs consisted of the same item and context word as those presented during the study list. Target-different word pairs consisted of the same item as that on the study list, paired with a new context word. Distractor-same word pairs consisted of a new word presented in green with a context word from the study list. Distractor-different word pairs comprised two new words that had not been previously presented on the study list. In addition, all the test categories were distributed equally between abstract ( 20 per category) and concrete ( 20 per category) word pairs.

\section{Results and Discussion}

Mean HR, FAR, and $d^{\prime}$ for Experiment 1 are presented in Table 2. HR minus FAR can be computed directly from the table. The CE column corresponds to the difference between the same- and the different-context conditions. CE effect sizes are presented in Table 5.

\section{HR}

A mixed factorial ANOVA conducted on the HR revealed significant main effects of word concreteness and context $[F(1,91)=19.67$ and $F(1,91)=48.85$, respectively; $p \mathrm{~s}<.05$,]. Generally, concrete words elicited a higher HR than did abstract words, and same contexts
Table 2

Experiment 1: Mean Hit Rates (HRs), False Alarm Rates (FARs), and $d^{\prime}$ Based on Encoding Instruction and Context

\begin{tabular}{|c|c|c|c|c|c|c|}
\hline \multirow[b]{3}{*}{ Encoding } & \multicolumn{6}{|c|}{ Word Concreteness } \\
\hline & \multicolumn{3}{|c|}{ Concrete } & \multicolumn{3}{|c|}{ Abstract } \\
\hline & $\mathrm{SC}$ & DC & $\mathrm{CE}$ & $\mathrm{SC}$ & DC & $\mathrm{CE}$ \\
\hline \multicolumn{7}{|l|}{ Imagery } \\
\hline HR & .72 & .46 & .26 & .58 & .41 & .17 \\
\hline FAR & .19 & .13 & .06 & .25 & .19 & .06 \\
\hline$d^{\prime}$ & 1.58 & 1.05 & 0.53 & 1.00 & 0.71 & 0.29 \\
\hline \multicolumn{7}{|l|}{ Rote } \\
\hline HR & .64 & .52 & .12 & .59 & .50 & .09 \\
\hline FAR & .28 & .22 & .06 & .31 & .27 & .04 \\
\hline$d^{\prime}$ & 1.09 & 0.89 & 0.20 & 0.86 & 0.71 & 0.15 \\
\hline \multicolumn{7}{|l|}{ Item only } \\
\hline HR & .60 & .57 & .03 & .53 & .54 & -.01 \\
\hline FAR & .26 & .22 & .04 & .27 & .26 & .01 \\
\hline$d^{\prime}$ & 1.02 & 1.03 & -0.01 & 0.84 & 0.83 & 0.01 \\
\hline
\end{tabular}

Note-SC, same context; DC, different context; CE, context effect (same-different).

resulted in a higher HR than did different contexts. These results are consistent with previous research on concreteness (e.g., Glanzer \& Adams, 1990) and context-dependent recognition (e.g., Bayen et al., 2000; Murnane et al., 1999). An encoding $\times$ context interaction was present $[F(2,91)=13.00, p<.05]$. It appears that the difference in HR between the same- and the different-context conditions was greater in the imagery condition than in the rote and item-only conditions. Furthermore, a word concreteness $\times$ context interaction was present $[F(1,91)=4.31$, $p<.05]$. The presence of this interaction is most likely due to a larger difference in the HR for the same than for the different context for the concrete words as opposed to the abstract words. Removing the item-only condition from the analyses did not change the presence of the main effects and interactions, nor did removal of the rote condition. However, removing the imagery condition and examining the interactions using only the rote and itemonly conditions revealed that the concreteness $\times$ context interaction was no longer significant. Thus, it appears that the difference in context as a function of concreteness for the imagery condition is driving the interaction, in comparison with the other encoding conditions.

In addition, planned comparisons with paired $t$ tests were utilized to examine our predictions regarding context-dependent recognition for the HR. We predicted that context-dependent recognition would be present for both the imagery and the rote conditions and that it would not be present for the item-only condition. The data support these predictions. In the imagery condition, a significant difference between same and different context was present in the HRs for both concrete and abstract words $[t(31)=5.18$ and $t(31)=4.30, p \mathrm{~s}<.001$, respectively $]$. In addition, there was a significant difference between same context and different context in the HRs for both concrete and abstract words in the rote condition $[t(30)=$ 3.73 and $t(30)=3.78$, respectively; $p$ s $<.001]$. This is consistent with the notion that an associated context or ensemble increases the HR for same-context conditions, 
relative to different-context conditions. No effects were detected for concrete and abstract HR for the item-only condition.

\section{FAR}

An ANOVA conducted on the FAR revealed the presence of two main effects: Both word concreteness and context affected the FAR $[F(1,91)=8.12$ and $F(1,91)=$ 22.26 , respectively; $p$ s $<.05$ ]. Overall, the same-context conditions resulted in a larger FAR than did the differentcontext conditions, and the abstract words resulted a in larger FAR than did the concrete words. Again, these results support prior research in which same context produced larger FARs (Murnane et al., 1999), and abstract words produced larger FARs than did concrete words, as indicated by the concreteness mirror effect (Glanzer \& Adams, 1990). No interactions were present.

As with the HR data, we conducted paired $t$-test comparisons to detect the presence of context-dependent recognition for the FAR. We predicted that context-dependent recognition would occur in the rote condition, would be unlikely to occur in the imagery condition, and should not occur in item-only condition. The imagery condition revealed context-dependent recognition for the FAR for both the concrete and the abstract words $[t(31)=3.20$, $p=.003$, and $t(31)=2.72, p=.01$, respectively]. However, both the rote and the item-only conditions failed to demonstrate context-dependent recognition for the FAR.

\section{Discrimination}

A mixed factorial ANOVA on $d^{\prime}$ was conducted to examine whether our manipulations had differential effects on context-dependent discrimination $\left(d^{\prime}\right)$. In this analysis, the two-way and three-way interactions are of interest, since these interactions reveal whether our manipulations affected the degree to which test context affects discrimination. In addition to predicting a three-way interaction of encoding, concreteness, and context, we expected that we would find an encoding $\times$ context interaction and an encoding $\times$ concreteness interaction. An interaction of encoding and context was present $[F(2,91)=5.05, p<$ $.05]$. This interaction might be explained by the imagery and rote conditions, particularly the imagery condition, which demonstrated larger differences in $d^{\prime}$ for samecontext than for different-context conditions; this pattern was not observed in the item-only condition. An inspection of Table 2 reveals that context had a greater impact for the imagery and rote conditions. This interaction was still present when the item-only condition was removed from the analysis, reflecting the fact that $d^{\prime}$ was affected differently for the imagery condition than for the rote condition.

No other interactions were significant. We had expected that the imagery condition would result in an interaction of concreteness and context, but analyses revealed that neither the imagery nor the other two encoding conditions produced such an interaction. In addition, the results indicated that there was a main effect of both word concreteness and context $[F(1,91)=31.12$ and $F(1,91)=13.62$, respectively, $p \mathrm{~s}<.05]$.
We also predicted that context-dependent discrimination would be found only in the imagery condition and not in the rote or item-only condition. Planned comparisons using paired $t$ tests provided support for this prediction. In the imagery condition, context-dependent discrimination was present for both concrete and abstract words $[t(31)=$ $3.67, p=.001$, and $t(31)=2.27, p=.03$, respectively]. Despite the fact that the FAR produced CEs in the imagery condition, discrimination still occurred, since the HR CEs were substantially greater than the FAR CEs. Neither the rote nor the item-only condition revealed such an effect for either concrete or abstract words.

In sum, Experiment 1 revealed context-dependent recognition in HRs for both imagery and rote rehearsal conditions and context-dependent discrimination only for the imagery condition. No such CEs were revealed for the item-only condition. Interestingly, if we examine the differences between same and different contexts for $d^{\prime}$, it is evident that some degree of context-dependent discrimination is present for both the imagery and the rote conditions. However, the magnitude of the differences is larger for the imagery condition than for the rote condition. In fact, examination of the CE sizes, presented in Table 5 using Cohen's $d$, yielded the largest effect sizes for imagery and somewhat smaller effect sizes for the rote condition. In contrast, the effect sizes for the item-only condition were virtually zero. Given our observed effect sizes, it is possible that our failure to detect context-dependent discrimination in the rote condition might have been the result of low statistical power.

\section{EXPERIMENT 2}

Experiment 2 was designed to increase our power by increasing the number of participants in the experiment, in addition to slightly decreasing the list length to 60 word pairs (30 concrete and 30 abstract) during the study session. The decrease in the number of pairs was designed to ensure that the previous results were not the result of HR floor effects due to list length, thus making the interaction difficult to detect in Experiment 1.

\section{Method}

\section{Participants}

One hundred fifty undergraduates at the University of Maryland participated in the experiment in order to fulfill partial course requirements.

\section{Materials}

Study and test lists were constructed in the same manner as in the previous experiment, with abstract and concrete word pairs selected from the Toronto Word Pool (Friendly et al., 1982). Word pairs appeared on the screen with the left word in green text (target word) and the right word in black text (context word). All the word pairs were presented in the middle of the screen in 28-point font. The pairs were always presented as an intact pair in terms of concreteness.

\section{Design and Procedure}

The experiment consisted of a 2 (concreteness: abstract or concrete) $\times 3$ (encoding: rote, imagery, or item only) $\times 2$ (test context: same or different) design. Word concreteness and test context was 
manipulated within participants, and encoding was manipulated between participants.

Study phase. Sixty word pairs ( 30 concrete and 30 abstract) were randomly presented on a computer screen for a 3 -sec duration. The participants were randomly assigned to one of three encoding instructions conditions. In the rote condition, the participants were instructed to repeat both words in the word pair over and over (subvocally) until the words disappeared from the screen. In the imagery condition, the participants were instructed that they should integrate both words into a vivid image. In the item-only condition, the participants were instructed to repeat once (subvocally) the word that appeared in green. All the participants were told that their memory for the words that appeared in green would be tested at a later time during the experiment.

Test phase. The participants were randomly presented with 120 word pairs, with the left word of the pair appearing in green text and right word in black text. As in the previous experiment, the participants were asked to determine whether the word appearing in green was on the study list. If the word was on the list, they were instructed to press the " $T$ " key on the keyboard, and if the word was not on the study list they were instructed to press the "F" key. The test word pairs were distributed into the following categories: 30 target same, 30 target different, 30 distractor same, and 30 distractor different, constructed in the same manner as that described in the previous experiment. In addition, all the categories were divided into an equal number of abstract ( 15 per category) and concrete ( 15 per category) word pairs.

\section{Results and Discussion}

Mean HR, FAR, and $d^{\prime}$ for Experiment 2 are presented in Table 3. HR minus FAR can be computed directly from the table, and the CE column corresponds to the difference between same- and different-context conditions. CE effect sizes are presented in Table 5.

\section{HR}

A mixed factorial ANOVA conducted on HR revealed main effects of word concreteness and context $[F(1,147)=$ 5.53 and $F(1,147)=69.06$, respectively; $p$ s $<.05]$. Like the pattern demonstrated in Experiment 1, same-context HR was larger than different-context HR, and concrete HR was greater than abstract HR. In addition, there was a main effect of encoding that was not present in the previous experiment $[F(2,147)=3.85, p<.05]$. The presence of the encoding main effect was most likely due to the increased HR for both the same- and the differentcontext conditions in the item-only condition, which, most likely, was the result of the shorter list length. When the item-only condition was removed from the analysis, the main effect of encoding was no longer significant. As in the previous experiment, two interactions were present: context $\times$ encoding and context $\times$ word concreteness $[F(2,147)=11.04$ and $F(1,147)=5.14$, respectively; $p$ s $<.05]$. The context $\times$ encoding interaction reflects the pattern that the HR is larger for the same context than the different context for both the rote and the imagery conditions, but not for the item-only condition. The context $\times$ concreteness interaction effect on the HR might be the result of differences between concrete and abstract words for same-context conditions, but small, negligible differences between concreteness for different-context conditions. In contrast to Experiment 1, these interactions were
Table 3

Experiment 2: Mean Hit Rates (HRs), False Alarm Rates (FARs), and $d^{\prime}$ Based on Encoding Instruction and Context

\begin{tabular}{lrrrrrrr}
\hline & \multicolumn{5}{c}{ Word Concreteness } \\
\cline { 2 - 4 } \cline { 6 - 7 } Encoding & SC & DC & CE & & SC & DC & CE \\
\cline { 2 - 4 } Imagery & & & & & & \\
$\quad$ HR & .68 & .46 & .22 & .62 & .45 & .17 \\
FAR & .14 & .09 & .05 & .23 & .18 & .05 \\
$\quad d^{\prime}$ & 1.67 & 1.19 & 0.48 & & 1.18 & 0.85 & 0.33 \\
Rote & & & & & & \\
$\quad$ HR & .67 & .53 & .14 & .63 & .53 & .10 \\
FAR & .23 & .17 & .06 & .28 & .26 & .02 \\
$d^{\prime}$ & 1.33 & 1.13 & 0.20 & 1.03 & 0.81 & 0.22 \\
Item only & & & & & & \\
$\quad$ HR & .68 & .63 & .05 & .63 & .62 & .01 \\
FAR & .15 & .15 & .00 & .20 & .21 & -.01 \\
$d^{\prime}$ & 1.63 & 1.47 & 0.16 & 1.32 & 1.24 & 0.08 \\
\hline
\end{tabular}

Note-SC, same context; DC, different context; CE, context effect.

no longer significant when the item-only condition was removed from the analyses.

Paired comparisons were conducted on HRs to reveal context-dependent recognition. Again, we predicted that context-dependent recognition would be present in both the imagery and the rote conditions, but not in the itemonly condition. As was predicted, context-dependent recognition was present in the imagery condition for HRs for both concrete and abstract words $[t(49)=5.68, p<.001$, and $t(49)=5.86, p<.001$, respectively]. A similar pattern existed in the rote condition for the HR, where there was a significant difference for the HR for concrete and abstract words, $[t(49)=4.64, p<.001$, and $t(49)=4.04$, $p<.001$, respectively]. However, the item-only condition was not consistent with the previous experiment, since for Experiment 2 context-dependent recognition was present for concrete words $[t(49)=2.17, p=.04]$. Note that the general pattern is consistent with that in Experiment 1, in that the HR is higher in the same-context condition than in the different-context condition for both imagery and rote conditions, yet the item-only condition produced mixed results by producing context-dependent recognition in this experiment for concrete words.

\section{FAR}

Regarding the FAR, consistent with the HR data for this experiment, all the main effects were statistically significant. There was a main effect of concreteness, with the FAR higher for abstract words $[F(1,147)=59.51, p<$ .05]. The main effect of context reflects the fact that the FAR was greater under same-context than under differentcontext conditions $[F(1,147)=12.68, p<.05]$. In addition, there was a main effect of encoding $[F(2,147)=$ $7.00, p<.05]$, with the rote condition producing a larger FAR than did the imagery and item-only conditions. No interactions were present for the FAR.

Planned comparisons on the FAR, using paired $t$ tests, revealed context-dependent recognition. As in Experiment 1 , context-dependent recognition for both concrete and abstract words was found in the imagery condition 
$[t(49)=3.09, p=.003$, and $t(49)=2.13, p=.04$, respectively]. In addition, the rote condition demonstrated a context-dependent recognition effect of FAR, but only for concrete words $[t(49)=2.64, p=.01]$. This pattern differs from that in Experiment 1, in that there was no effect of context on FARs in the rote condition. We had expected that there would be context-dependent recognition for the rote condition, and an inspection of Table 3 reveals that the FARs for the same-context condition, in comparison with the different-context condition, for concrete and abstract words were in the predicted direction.

\section{Discrimination}

Again, we expected to find a three-way interaction of encoding, concreteness, and context, as well as encoding $\times$ context and encoding $\times$ concreteness interactions. The interaction of context and encoding condition was present $[F(2,147)=2.95, p=.05]$. Consistent with Experiment 1 , the imagery and rote conditions, particularly the imagery condition, demonstrated larger differences in $d^{\prime}$ in the same-context condition than in the differentcontext condition. An inspection of Table 3 indicates that test context had a greater impact on $d^{\prime}$ for the imagery than for the rote condition.

However, unlike in the previous experiment, the itemonly condition for concrete words also revealed a CE. When the item-only condition was removed from these analyses, the interaction was no longer present. No other interactions were significant. We had expected that there would be a context $\times$ concreteness interaction for the imagery condition, but not for the other encoding conditions. However, separate analyses on each of the encoding conditions failed to reveal the interaction in any of the encoding conditions. In addition, the results indicated that there was a main effect of both word concreteness and context $[F(1,147)=59.13$ and $F(1,147)=24.80$, respectively; $p \mathrm{~s}<.05]$. In addition, there was a main effect of encoding $[F(2,147)=5.74, p<.05]$; however, removing the itemonly condition from the analyses resulted in the encoding main effect's no longer being significant.

Planned comparisons using paired $t$ tests were used to test for context-dependent discrimination. As was expected, the imagery condition resulted in context-dependent discrimination for both concrete and abstract words $[t(49)=3.89$, $p=.0003$, and $t(49)=2.92, p=.005$, respectively]. However, the rote condition also resulted in context-dependent discrimination, but only for abstract words $[t(49)=2.30$, $p=.03]$. This effect was not produced in the first experiment. As was expected, the item-only condition did not demonstrate context-dependent discrimination for either concrete or abstract words.

In sum, Experiment 2 revealed context-dependent recognition for both imagery and rote rehearsal conditions. However, in contrast to the first experiment, we found context-dependent discrimination for both the imagery and the rote rehearsal conditions. We also found contextdependent recognition for concrete words in the item-only rehearsal condition, but no evidence of context-dependent discrimination.
As before, we calculated effect sizes for contextdependent discrimination as a function of encoding and word concreteness, and these values are presented in Table 5. Notably, the effect size was largest for the imagery condition, with concrete pairs having a greater effect than did abstract pairs. This pattern is consistent with that in Experiment 1. Thus, although the abstract pairs had fewer imaginal properties (as reflected in imaginability ratings in word norms), the fact that context discrimination exists indicates that the interactive imagery encoding instruction was successful even when the words were relatively abstract. However, concrete pairs seem to have had an advantage, in comparison with abstract pairs, but only for the imagery condition. This is consistent with previous findings that it is possible to form images for abstract pairs. ${ }^{6}$ Although the effect sizes indicate that there was a difference between concrete and abstract words in the imagery condition, individual analyses indicated that there was not enough power to detect this difference in the effect sizes. Another point of interest is the fact that a medium effect was present for the rote condition. Thus, rote rehearsal can potentially lead to context-dependent discrimination, but the magnitude of the effect is much smaller than when interactive imagery is used. Furthermore, a small effect was present for the item-only condition, with a slightly greater advantage for concrete words. The overall pattern of effect sizes is consistent with our predictions regarding the pattern of context-dependent discrimination, in that effect size in the imagery condition would be greater than that in the rote condition and that in the rote condition would be greater than or equal to that in the item-only condition.

\section{EXPERIMENT 3}

Experiment 3 was designed to examine whether the context-dependent discrimination observed in the rote condition in Experiment 2 was a result of using a unique context word per item word at study. Our concern was that there was the potential for the participants to use the unique context words as a cue at test for item word recognition, which, in turn, could enhance context-dependent discrimination for same- versus different-context conditions (cf. Bjork \& Richardson-Klavehn, 1989; Smith \& Vela, 2001), thus creating a condition similar to the ensemble-only condition where context-dependent discrimination can occur.

In order to ensure that the context-dependent discrimination for the rote condition was not due to the use of a unique context word each time, we used the same context word paired with multiple targets during study. Using multiple contexts places the imagery condition in the last row of Table 1, where context-dependent recognition should occur for HRs and FARs, and context-dependent discrimination is likely to occur. We expected that the rote condition would still correspond to the second row of Table 1. Thus, context-dependent recognition should occur, and contextdependent discrimination would be unlikely to occur. In addition, we did not include the item-only condition from the previous experiments, since we did not anticipate that 
this condition would show reliable context-dependent discrimination, as demonstrated in the previous experiments.

\section{Method}

\section{Participants}

One hundred sixteen undergraduates at the University of Maryland participated in the experiment in order to fulfill partial course requirements.

\section{Materials}

As in the previous experiments, study and test lists were composed of abstract and concrete word pairs selected from the Toronto Word Pool (Friendly et al., 1982). Unlike in the previous experiments, the same context word was paired multiple times with target words. Specifically, an identical context word appeared six times with 6 unique target words. Thus, in this experiment, a total of 10 words served as context words ( 5 for abstract targets and 5 for concrete targets). As before, word pairs were presented in 28-point font in the center of the screen, with the left word in green text (target word) and the right word in black text (context word). The words in the pair consisted of either concrete or abstract pairs.

\section{Design and Procedure}

The experiment consisted of a 2 (concreteness: abstract or concrete) $\times 2$ (encoding: rote or imagery) $\times 2$ (test context: same or different) design. Encoding was manipulated between participants, and word concreteness and test context were manipulated within participants.

Study phase. The study phase consisted of a total of 60 word pairs ( 30 concrete and 30 abstract). Each word pair was presented for $3 \mathrm{sec}$ in a random order. The participants were randomly assigned to either the rote or the imagery encoding condition. As in the previous experiments, the participants in the rote condition were instructed to repeat both words in the pair over and over (subvocally) until the words disappeared from the screen. In the imagery condition, the participants were instructed that they should form a vivid mental picture of the things named by the words interacting with each other. All the participants were informed that at a later time, their memory for the words that appeared in green would be tested.

Test phase. During the test phase, the participants were randomly presented with 120 word pairs (30 target same, 30 target different, 30 distractor same, and 30 distractor different), with an equal number of abstract and concrete pairs per pair category. The participants were asked to determine whether the word appearing in green was on the study list. If the word was on the list, they were instructed to press the "A" key on the keyboard, and if the word was not on the study list, they were instructed to press the "L" key. (The "A" and "L" keys were counterbalanced.)

\section{Results and Discussion}

Mean HRs, FARs, and $d^{\prime}$ for Experiment 3 are presented in Table 4. HR minus FAR can be computed directly from the table, and the CE column corresponds to difference between same- and different-context conditions. CE effect sizes are presented in Table 5 .

\section{HR}

A mixed factorial ANOVA was conducted to examine mean HR as a function of word concreteness and encoding instructions. The results reveal a main effect of encoding instruction $[F(1,114)=4.36, p<.05]$, indicating that the imagery condition resulted in a greater number of hits than did the rote condition. In addition, there were main effects of word concreteness and context $[F(1,114)=19.41$ and $F(1,114)=92.61$, respectively; $p$ s $<.05]$. Similar to pre-
Table 4

Experiment 3: Mean Hit Rates (HRs), False Alarm Rates (FARs), and $d^{\prime}$ Based on Encoding Instruction and Context

\begin{tabular}{|c|c|c|c|c|c|c|}
\hline \multirow[b]{3}{*}{ Encoding } & \multicolumn{6}{|c|}{ Word Concreteness } \\
\hline & \multicolumn{3}{|c|}{ Concrete } & \multicolumn{3}{|c|}{ Abstract } \\
\hline & $\mathrm{SC}$ & $\mathrm{DC}$ & $\mathrm{CE}$ & $\mathrm{SC}$ & DC & $\mathrm{CE}$ \\
\hline \multicolumn{7}{|l|}{ Imagery } \\
\hline HR & .76 & .57 & .19 & .64 & .49 & .15 \\
\hline FAR & .13 & .09 & .04 & .22 & .16 & .06 \\
\hline$d^{\prime}$ & 2.03 & 1.60 & 0.43 & 1.27 & 1.06 & 0.21 \\
\hline \multicolumn{7}{|l|}{ Rote } \\
\hline HR & .66 & .49 & .17 & .62 & .45 & .17 \\
\hline FAR & .18 & .12 & .06 & .26 & .18 & .08 \\
\hline$d^{\prime}$ & 1.47 & 1.21 & 0.26 & 1.10 & 0.86 & 0.24 \\
\hline
\end{tabular}

Note-SC, same context; DC, different context; CE, context effect.

vious results, there were more hits for concrete than for abstract words, and the same-context condition resulted in a greater number of hits than did the different-context condition. Unlike in the previous experiments, there was an interaction between word concreteness and encoding instructions $[F(1,114)=3.97, p<.05]$. The interaction was due to the difference in HR for the concrete condition under imagery instructions, in comparison with the abstract condition, as well as for both the concrete and the abstract conditions under rote instructions. The latter three cells are similar in terms of hits, in comparison with the concrete imagery condition. None of the other interactions was significant.

Planned comparisons were conducted to examine context-dependent recognition. Under imagery instructions, both concrete and abstract words resulted in context-dependent recognition $[t(57)=5.34$ and $t(57)=$ 5.71, respectively; $p$ s $<.01]$. This pattern was also present under rote instructions for both concrete and abstract words $[t(57)=5.31$ and $t(57)=6.04, p \mathrm{~s}<.01]$.

\section{FAR}

A mixed factorial ANOVA conducted on mean FAR revealed only main effects of word concreteness and context $[F(1,114)=64.50$ and $F(1,114)=50.12$, respectively;

Table 5

Effect Size (Cohen's $d$ ) for Context Effects (Same-Different) Based on Encoding Instructions and Word Type

\begin{tabular}{|c|c|c|c|c|c|c|}
\hline \multirow[b]{3}{*}{ Condition } & \multicolumn{6}{|c|}{ Word Concreteness } \\
\hline & \multicolumn{3}{|c|}{ Concrete } & \multicolumn{3}{|c|}{ Abstract } \\
\hline & HR & FAR & $d^{\prime}$ & HR & FAR & $d^{\prime}$ \\
\hline \multicolumn{7}{|l|}{ Experiment 1} \\
\hline Imagery & 1.21 & 0.39 & 0.69 & 0.77 & 0.36 & 0.52 \\
\hline Rote & 0.63 & 0.31 & 0.27 & 0.56 & 0.23 & 0.27 \\
\hline Item only & 0.15 & 0.25 & -0.02 & -0.05 & 0.05 & 0.02 \\
\hline \multicolumn{7}{|l|}{ Experiment 2} \\
\hline Imagery & 0.96 & 0.55 & 0.64 & 0.85 & 0.32 & 0.53 \\
\hline Rote & 0.66 & 0.37 & 0.26 & 0.50 & 0.13 & 0.30 \\
\hline Item only & 0.31 & 0 & 0.27 & 0.05 & -0.07 & 0.13 \\
\hline \multicolumn{7}{|l|}{ Experiment 3} \\
\hline Imagery & 0.90 & 0.41 & 0.52 & 0.71 & 0.45 & 0.31 \\
\hline Rote & 0.91 & 0.53 & 0.41 & 0.79 & 0.57 & 0.39 \\
\hline
\end{tabular}

Note-HR, hit rate; FAR, false alarm rate. 
$p \mathrm{~s}<.05]$. As in the previous experiments, there were more false alarms in the same-context condition than in the different-context condition, as well as more false alarms for abstract words than for concrete words. There was no effect of encoding instructions on FAR, and none of the interactions was significant.

Planned comparisons conducted on context-dependent recognition for the FAR revealed the same pattern as with the HR, whereby both imagery and rote instructions resulted in context-dependent recognition for both concrete and abstract words. Under imagery instructions, concrete and abstract words were significant $[t(57)=3.26$ and $t(57)=3.28, p$ s $<.01]$. A similar pattern was also found under rote instructions for concrete and abstract words $[t(57)=3.96$ and $t(57)=4.02, p$ s $<.01]$. Unlike in the first two experiments, reliable CEs were observed for both concrete and abstract words.

\section{Discrimination}

A mixed factorial ANOVA was conducted on mean $d^{\prime}$ based on encoding instructions and word concreteness. The results indicated a main effect of encoding instructions $[F(1,114)=12.74, p<.05]$, whereby the imagery condition had a larger $d^{\prime}$ than did the rote condition. In addition, there were main effects of word concreteness and context $[F(1,114)=81.39$ and $F(1,114)=28.78$, respectively; $p \mathrm{~s}<.05]$. These main effects were reflected in overall greater discrimination for concrete words than for abstract words, as well as greater discrimination under the same context than under a different context. However, these main effects were qualified by a significant word concreteness $\times$ encoding instruction interaction $[F(1,114)=6.38, p<.05]$. As is clear from examining the magnitude of the CEs on $d^{\prime}$ in 4 , only the concrete words benefited from the imagery instructions. There was no difference in size of the CE on $d^{\prime}$ between abstract and concrete words for the rote rehearsal condition or between rote and imagery condition for the abstract words.

Note, however, that there were significant differences between same and different context on $d^{\prime}$ for both abstract and concrete words under both imagery and rote rehearsal instructions. Planned comparisons revealed significant effects under imagery instructions for concrete words $[t(57)=3.32, p<.01]$ and abstract words $[t(57)=2.44$, $p=.02]$ and under rote instructions for concrete words $[t(57)=2.90, p<.01]$ and abstract words $[t(57)=2.49$, $p=.02]$. This finding indicates that context-dependent discrimination is possible in the rote condition and that it is not an artifact of using a unique context word.

\section{Unique Versus Common Contexts}

One interesting question is whether the CEs observed in Experiment 3 are similar in magnitude to those observed in Experiments 1 and 2. Recall that in Experiments 1 and 2 , each item was studied in a unique context, whereas in Experiment 3, multiple items were studied in a single context; otherwise, the experiments were essentially identical (except for slight differences in the lengths of the study lists) in how they were carried out and the instructions provided to the participants. An inspection of the effect sizes in Table 5 raises the possibility that the size of the $\mathrm{CE}$ for the rote condition was larger in Experiment 3 than in Experiments 1 and 2. This would be an interesting finding, if supported statistically, because it would suggest that the uniqueness of the context affects the magnitude of the CE. We examined this statistically by conducting cross-experiment analyses to compare the magnitude of context-dependent discrimination. The analyses were done separately for the rote rehearsal and imagery conditions. For the participants given rote rehearsal instructions, there were no differences between experiments in the magnitude of context-dependent discrimination, as measured by $d^{\prime}[F(2,136)=0.23, p>.05]$, nor did word concreteness affect the magnitude of context-dependent discrimination $[F(1,136)=0.03, p>.05]$. That there were no differences between the three experiments suggests that the significant context-dependent discrimination effect found in Experiment 3 was likely due to its having greater statistical power.

An inspection of Table 5 also suggests that the CE effects in the imagery conditions appear to be smaller for Experiment 3 than for Experiments 1 and 2. However, as was the case for the rote condition, cross-experiment analyses failed to reveal a significant difference between experiments on the magnitude of the CE for $d^{\prime}[F(1,137)=$ $0.32, p>.05]$. However, there was a reliable effect of word concreteness $[F(1,137)=4.91, p=.03]$. Thus, although the magnitude of the effect appears somewhat attenuated in the last experiment, the effect was reliable across all the experiments, regardless of whether unique or multiple contexts were used.

\section{GENERAL DISCUSSION}

The three experiments presented in this article reveal a relatively consistent pattern of results. Consistent with ICE predictions, HR was substantially greater in the same context than in the different context for the conditions in which people were instructed to use both item and context information (namely, the imagery and rote conditions). Context-dependent recognition occurred for both concrete and abstract words, but only when the participants engaged in imagery or rote rehearsal that required the use of both item and context information. The finding that context-dependent recognition was observed for verbal context is consistent with earlier research findings (Light \& Carter-Sobell, 1970; Tulving \& Thomson, 1973).

A different pattern emerged for the FAR in contextdependent recognition. Imagery instructions produced context-dependent recognition for both concrete and abstract words across all the experiments. In terms of ICE predictions, CE should not occur for the FAR when unique contexts are used (as indicated in the third row of Table 1). Because the CE effect for the HR was substantially greater than the CE for the FAR, a net discrimination effect was still detected. Furthermore, in terms of FAR, there were inconsistencies for the rote condition. For example, contextdependent recognition was observed for FAR for concrete 
words in Experiment 2 and for both concrete and abstract words in Experiment 3. According to ICE, we expected CEs to occur for FAR for both concrete and abstract words in all three experiments.

In terms of context-dependent discrimination, the imagery condition produced a consistent pattern whereby context-dependent discrimination was observed in all three experiments for both concrete and abstract words, although the effect was smaller with abstract words than with concrete words. A cross-experiment analysis revealed that this pattern held for all three experiments, despite the slightly lower CE effects found in Experiment 3 than in Experiments 1 and 2. The rote condition resulted in context-dependent discrimination for abstract words (Experiment 2) when unique-context words were used and for both concrete and abstract words (Experiment 3) when same-context words were used multiple times. Although discrimination was of the same magnitude across all three experiments for the rote conditions, the effect was not significant for either the concrete or the abstract words in Experiment 1 and was not significant for the concrete words in Experiment 2.

Our results partially support the hypothesis that ICE theory can be applied to verbal contexts. On the one hand, our results clearly showed that interactive imagery instructions are sufficient to produce context-dependent discrimination and that this instruction is somewhat robust across word concreteness. Interestingly, our effect sizes were greater than those reported by Rutherford (2004), who combined global and local context manipulations. However, the use of word pairs is most likely the reason for this difference.

Also consistent with ICE are our findings that item-only instructions consistently failed to produce context-dependent discrimination and rarely produced context-dependent recognition (and then only for HR, using concrete words in Experiment 2). However, that rote rehearsal was successful at producing context-dependent discrimination might raise some problems for ICE theory. On the one hand, on the basis of a strict interpretation of ICE theory, one would presume that rote rehearsal instructions should not have led to context-dependent discrimination. This is because without explicit instructions to integrate item information with context information, no ensembles should have been formed and, therefore, context-dependent discrimination should not have occurred. However, that context-dependent discrimination occurred under rote rehearsal instructions suggests one of several possibilities.

One possible explanation is that rote rehearsal led to an association between the two words in the pair. Research investigating the mechanisms underlying incidental learning of word pairs under rote rehearsal has revealed that participants do form an association between the words in a pair. The robust finding is that intact pairs are better recognized than rearranged pairs (Dobbins, Kroll, \& Yonelinas, 2004; Nairne, 1983). It should be noted that our experiments diverge from associative research in several ways. First, our participants were intentionally instructed that there would be a memory test. Second, we explic- itly stated that the green word was the target word and asked the participants to repeat both words together. ${ }^{7}$ It seems unlikely that our paradigm would result in the same type of association that has been observed in incidental learning paradigms using rote rehearsal instructions. The presence of an association between the words would have revealed a pattern similar to associated context (see row 2 of Table 1). In this case, we would expect the same magnitude of HR and FAR CEs, so that discrimination would not occur. Furthermore, we would expect that word concreteness would affect both HR and FAR differentially (as observed in mirror effects based on concreteness; Glanzer et al., 1993). Lastly, we would expect that the associated context would produce smaller effects than the effects in the imagery condition. Although we observed smaller effects in the rote condition, the fact that we consistently do not observe a word concreteness effect in all three experiments leads us to believe that these results are not consistent with an associated context explanation.

Another possible explanation is that rote rehearsal led to ensemble formation. Clearly, ensembles can be based on nonvisual (e.g., phonological or semantic) information or even a simpler integration, such as the unification of the two words into one long word. In partial support of the possibility that rote rehearsal led to some form of nonimagery-based integration is the fact that the effect sizes for context-dependent discrimination for the imagery instructions were smaller for abstract words than for concrete words, whereas there were no such obvious differences in effect sizes for the rote rehearsal condition. However, if ensembles are based on nonimagery attributes, contextdependent discrimination should be independent of word concreteness. An interesting test of this might involve manipulating semantic relatedness or phonological similarity. If rote rehearsal leads to the formation of semantic or phonological ensembles, one should expect greater context-dependent discrimination when pairs are semantically related or phonologically similar. ${ }^{8}$

However, whether or not rote rehearsal led to the formation of nonimagery ensembles is unknown. Thus, whether or not the findings from the rote rehearsal condition can be taken as evidence against ICE is unclear. At the very minimum, however, that ensemble formation might be achieved via semantic or phonological integration suggests a few new avenues for exploring the factors that affect context-dependent discrimination.

How do our results fare with other theories of CEs? The cue overload hypothesis would predict that less discrimination should be observed when the context is used multiple times than when the verbal context is unique to the target (Watkins \& Watkins, 1975). Indeed, contextdependent discrimination decreased somewhat for the imagery condition in Experiment 3; however, the contextdependent discrimination still was sizable, and this effect was also obtained for the rote condition. Therefore, these results would not be in accord with the cue overload hypothesis.

The outshining hypothesis states that an environmental cue would be used when better cue sources are absent 
(Smith, 1988). Because of the nature of our manipulation of verbal context, incidental environmental cues would be ineffective because better cue sources (namely, the context word) are available. Interestingly, we found that pairing with the same-context cue (Experiment 3 ) resulted in larger magnitude context-dependent discrimination than did pairing with a unique-context cue (Experiments 1 and 2 ) for the rote conditions. This result is inconsistent with the outshining hypothesis, in that the same-context cue should not have produced better discrimination. However, these results are consistent with the integration hypothesis (Baddeley, 1982). In the integration hypothesis, CEs should occur when the item and the context are integrated in memory. This hypothesis is similar to ICE theory, except that the ICE theory distinguishes between integration (ensemble) and associated context (akin to Eich's, 1985, isolated imagery condition).

In sum, our research generally supports the hypothesis that ICE theory's predictions can be applied to verbal contexts. Our research replicates and extends the findings of Murnane et al. (1999), in that we found that contextdependent discrimination could be achieved using verbal context (rather than rich visual information) and that the effect was robust to word concreteness. That the rote rehearsal condition also produced context-dependent discrimination suggests that more needs to be done to examine the possibility that ensembles could be based on different attributes of the stimulus materials, such as the imaginal, phonological, or semantic attributes (Humphreys, 1976; Light \& Carter-Sobell, 1970). Such an investigation might be useful for delineating the conditions under which context-dependent discrimination is likely and unlikely to occur, as well as the conditions that produce the greatest amount of context-dependent discrimination.

\section{REFERENCES}

Baddeley, A. D. (1982). Domains of recollection. Psychological Review, 89, 708-729.

Bayen, U. J., Phelps, M. P., \& Spaniol, J. (2000). Age-related differences in the use of contextual information in recognition memory: A global matching approach. Journals of Gerontology: Psychological Sciences \& Social Sciences, 55B, P131-P141.

Bjork, R. A., \& Richardson-Klavehn, A. (1989). On the puzzling relationship between environmental context and human memory. In C. Izawa (Ed.), Current issues in cognitive processes: The Tulane Flowerree Symposium on Cognition (pp. 313-344). Hillsdale, NJ: Erlbaum.

Bradley, M. M., \& Glenberg, A. M. (1983). Strengthening associations: Duration, attention, or relations? Journal of Verbal Learning \& Verbal Behavior, 22, 650-666.

Bugelski, B. R. (1970). Words and things and images. American Psychologist, 25, 1002-1012.

Craik, F. I. M., Govoni, R., Naveh-Benjamin, M., \& Anderson, N. D. (1996). The effects of divided attention on encoding and retrieval processes in human memory. Journal of Experimental Psychology: General, 125, 159-180.

Craik, F. I. M., Naveh-Benjamin, M., Ishaik, G., \& Anderson, N. D. (2000). Divided attention during encoding and retrieval: Differential control effects? Journal of Experimental Psychology: Learning, Memory, \& Cognition, 26, 1744-1749.

Craik, F. I. M., \& Tulving, E. (1975). Depth of processing and the retention of words in episodic memory. Journal of Experimental Psychology: General, 104, 268-294.
DaLton, P. (1993). The role of stimulus familiarity in context-dependent recognition. Memory \& Cognition, 21, 223-234.

Dobbins, I. G., Kroll, N. E. A., \& Yonelinas, A. P. (2004). Dissociating familiarity from recollection using rote rehearsal. Memory \& Cognition, 32, 932-944.

EICH, E. (1985). Context, memory, and integrated item/context imagery. Journal of Experimental Psychology: Learning, Memory, \& Cognition, 11, 764-770.

Friendly, M., Franklin, P. E., Hoffman, D., \& Rubin, D. C. (1982). The Toronto Word Pool: Norms for imagery, concreteness, orthographic variables, and grammatical usage for 1,080 words. Behavior Research Methods \& Instrumentation, 14, 375-399.

Glanzer, M., \& Adams, J. K. (1990). The mirror effect in recognition memory: Data and theory. Journal of Experimental Psychology: Learning, Memory, \& Cognition, 16, 5-16.

Glanzer, M., Adams, J. K., Iverson, G. J., \& Kim, K. (1993). The regularities of recognition memory. Psychological Review, 100, 546-567.

Godden, D. R., \& Baddeley, A. D. (1975). Context-dependent memory in two natural environments: On land and underwater. British Journal of Psychology, 66, 325-331.

Groninger, L. D., \& GRoninger, L. K. (1982). Function of images in the encoding-retrieval process. Journal of Experimental Psychology: Learning, Memory, \& Cognition, 8, 353-358.

Hintzman, D. L. (1988). Judgments of frequency and recognition memory in multiple-trace memory model. Psychological Review, 95, 528-551.

HuMPHREYS, M. S. (1976). Relational information and the context effect in recognition memory. Memory \& Cognition, 4, 221-232.

Light, L. L., \& CARTER-SobelL, L. (1970). Effects of changed semantic context on recognition memory. Journal of Verbal Learning \& Verbal Behavior, 9, 1-11.

Murnane, K., \& Phelps, M. P. (1993). A global activation approach to the effect of changes in environmental context on recognition. Journal of Experimental Psychology: Learning, Memory, \& Cognition, 19, 882-894.

Murnane, K., \& Phelps, M. P. (1994). When does a different environmental context make a difference in recognition? A global activation model. Memory \& Cognition, 22, 584-590.

Murnane, K., \& PhelPs, M. P. (1995). Effects of changes in relative cue strength on context-dependent recognition. Journal of Experimental Psychology: Learning, Memory, \& Cognition, 21, 158-172.

Murnane, K., Phelps, M. P., \& Malmberg, K. (1999). Contextdependent recognition memory: The ICE theory. Journal of Experimental Psychology: General, 128, 403-415.

NAIRNE, J. S. (1983). Associative processing during rote rehearsal. Journal of Experimental Psychology: Learning, Memory, \& Cognition, 9, 3-20.

Richardson, J. T. E. (1998). The availability and effectiveness of reported mediators in associative learning: A historical review and an experimental investigation. Psychonomic Bulletin \& Review, 5, $597-$ 614.

RUTHERFORD, A. (2004). Environmental context-dependent recognition memory effects: An examination of ICE model and cue-overload hypotheses. Quarterly Journal of Experimental Psychology, 57A, 107-127.

Shiffrin, R. M., \& STEYvers, M. (1997). A model for recognition memory: REM - retrieving effectively from memory. Psychonomic Bulletin \& Review, 4, 145-166.

Sмiтh, S. M. (1988). Environmental context-dependent memory. In G. M. Davies \& D. M. Thomson (Eds.), Memory in context: Context in memory (pp. 13-34). Chichester, U.K.: Wiley.

Smith, S. M., Glenberg, A., \& BJork, R. A. (1978). Environmental context and human memory. Memory \& Cognition, 6, 342-353.

Smith, S. M., \& Vela, E. (2001). Environmental context-dependent memory: A review and meta-analysis. Psychonomic Bulletin \& Review, 8, 203-220.

Tulving, E., \& Thomson, D. M. (1973). Encoding specificity and retrieval processes in episodic memory. Psychological Review, 80, 353-370.

WatKInS, O. C., \& WatKInS, M. J. (1975). Buildup of proactive inhibition as a cue-overload effect. Journal of Experimental Psychology: Human Learning \& Memory, 104, 442-452. 
WiCKENS, D. D. (1987). The dual meanings of context: Implications for research, theory, and applications. In D. S. Gorfein \& R. R. Hoffman (Eds.), Memory and learning: The Ebbinghaus Centennial Conference (pp. 135-152). Hillsdale, NJ: Erlbaum.

\section{NOTES}

1. There is disagreement as to whether manipulating backgrounds on a screen can be construed as an environmental context. Murnane and Phelps (1993, 1994, 1995) used background as an environmental context; but see Smith and Vela (2001) for an alternative viewpoint.

2. The ICE model is a generalized global-matching model that is not wedded to specific representational or retrieval assumptions. As such, several of the existing global-matching models, such as MINERVA 2 (Hintzman, 1988) and REM (Shiffrin \& Steyvers, 1997), could be adopted as specific instantiations of ICE. However, since all of these models make essentially the same predictions, aside from differences that might arise because of different representational assumptions or different activation functions, tests of the ICE model's predictions do not shed light on any of these models in particular. Our goal in this research was not to test ICE per se but to examine the factors that affect contextdependent discrimination.

3. Although Murnane et al. (1999) allowed for the possibility that item and associated context alone will produce context-dependent discrimination, they argued that it is unlikely to occur.

4. Despite Eich's (1985) findings that the integrated imagery instructions were recognized slightly less than isolated imagery instructions in an environmental context design, we anticipate that interactive imagery instructions should result in better recognition in verbal contexts (cf. Craik \& Tulving, 1975).

5. Although the item-only condition does not preclude that the participants would not use the context word, they should be less likely to do so, since the instructions specifically stated that they would be tested for the word that appeared in green (the item-only word).

6 . We have found some evidence that imagery instructions are effective for abstract pairs during the allotted time, despite the fact that some researchers (e.g., Bugelski, 1970) have questioned the quality of the image under short presentation times. If the imagery participants could not form some sort of an image, we would expect that their performance would be identical to that in the rote condition for abstract words, and this is clearly not the case.

7. Because our primary goal was to understand $\mathrm{CE}$ in verbal context, using intact versus rearranged pairs would reveal that some form of an association had been made for the words in the pair, but not whether this association was based on context information.

8. Another potential explanation for the rote rehearsal pattern is that the rote participants did not follow our instructions to use rote rehearsal. Although one cannot rule out the possibility that the participants were not following the instructions, the pattern of results suggests that whatever the participants were doing in the rote condition, it was distinct from what was being done in the imagery and item-only conditions - and also consistent across experiments. For example, the participants in the imagery condition were affected by word concreteness, whereas the participants in the rote condition were not. In contrast, in comparison with the participants in the rote condition, who consistently showed CEs in Experiments 1 and 2, the participants in the item-only condition did not consistently show CEs. Taken together, the pattern of data suggests that our instructions to repeat the pair of words over and over led the participants to encode the item-context word pair in a manner that was distinctly different from how the item-context pair was encoded for the imagery and item-only conditions.

(Manuscript received October 10, 2002; revision accepted for publication May 31,2005 .) 